\title{
Self-shielding clumps in starburst clusters
}

\author{
Jan Palouš ${ }^{1}$, Richard Wünsch ${ }^{1}$, Soňa Ehlerová ${ }^{1}$ \\ and Guillermo Tenorio-Tagle ${ }^{2}$ \\ ${ }^{1}$ Astronomical Institute of the CAS, \\ Boční II 1401, 14131 Prague 4, Czech Republic \\ email: palous@ig.cas.cz \\ ${ }^{2}$ Instituto Nacional de Astrofísica Optica y Electronica, \\ AP 51, 72000 Puebla, Mexico \\ email: guillermotenoriotagle@gmail.com
}

\begin{abstract}
Young and massive star clusters above a critical mass form thermally unstable clumps reducing locally the temperature and pressure of the hot $10^{7} \mathrm{~K}$ cluster wind. The matter reinserted by stars, and mass loaded in interactions with pristine gas and from evaporating circumstellar disks, accumulate on clumps that are ionized with photons produced by massive stars. We discuss if they may become self-shielded when they reach the central part of the cluster, or even before it, during their free fall to the cluster center. Here we explore the importance of heating efficiency of stellar winds.
\end{abstract}

Keywords. Globular clusters: general, Stars: formation, Hydrodynamics

\section{Introduction}

High-precision photometry with Hubble Space Telescope and stellar spectroscopy with Very Large Telescope discovered multiple stellar generations in globular clusters: enhanced He content in blue main-sequences and anticorrelation of $\mathrm{Na}$ - $\mathrm{O}$ and other products of hydrogen burning in cluster stars, see Hempel et al. (2014), Gratton et al. (2012) and Poitto et al. (2012). It is assumed that the first stellar generation mixed its nuclear burning products with original gas preparing the medium for formation of further stellar generations. Possible explanations include slow winds of fast rotating massive stars (Krause et al. 2013), or winds of AGB stars (D'Ercole et al. 2012). We propose the cooling winds of massive stars as the place where the enriched stellar generation forms. Here we discuss when thermally unstable clumps self-shield the UV photons produced by young stars of the cluster to become seeds for secondary star formation.

\section{Cooling Winds}

In clusters above a critical mass, thermally unstable clumps are created out of hot gas of fast winds of massive stars (Tenorio-Tagle et al. 2007, Wünsch et al. 2011, Palouš et al. 2013, and references therein). They shrink to a smaller volume, and the surrounding hot cluster wind is not able to push them out from the cluster, on the contrary, under the influence of the cluster gravity, they stream down towards the cluster center (see Fig. 1). We derive the minimum time that is needed to accumulate enough mass so that the stream is self-shielded against UV photons from the star cluster. However, when the free fall time of clumps is shorter compared to the self-shielding time, the streams of thermally unstable clumps meet in the cluster center forming central concentration of warm gas. As soon as this central mass concentration is self-shielded, it cools further down to become the seed of a new enriched stellar generation. 

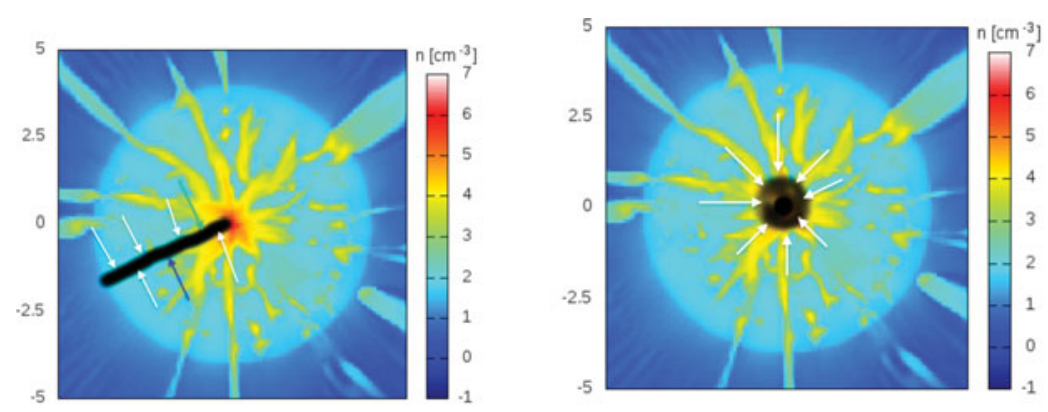

Figure 1. Left: stream of warm gas falling to the cluster center; Right: central condensation of warm gas.
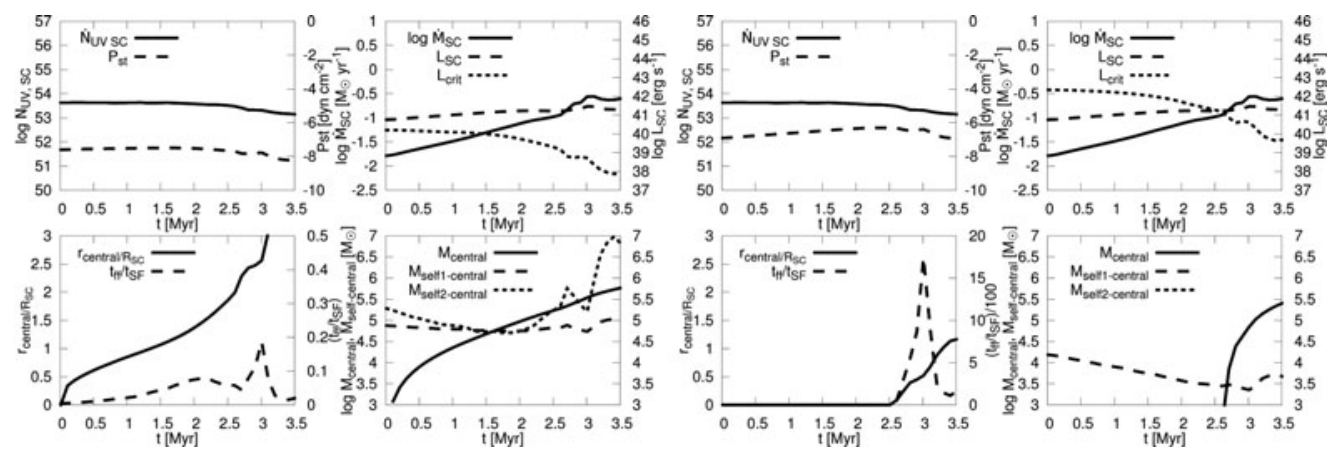

Figure 2. The time evolution of the UV photon flux $\dot{N}_{U V, S W C}$, pressure in the cluster wind $P_{s t}$, total mass flux $\dot{M}_{S C}$ and mechanical energy flux $L_{S C}$ in the cluster wind, radius of the central clump $r_{\text {central }}$, self-shielding time of the gas streams $t_{S F}$ and central mass concentration $M_{\text {central }}$ during the first 3.5 Myr. Left: $5 \%$ heating efficiency; Right $30 \%$ heating efficiency.

\section{Results}

The results of our semi-analytical calculations of the evolution of clusters during its first 3.5 Myr are given in Fig. 2. We assume a first stellar generation with the total mass $10^{7}$ $\mathrm{M}_{\odot}$ and a radius $3 \mathrm{pc}$. In such cluster, its wind is thermally unstable forming after $3.5 \mathrm{Myr}$ clumps with a total mass of a few times $10^{5} \mathrm{M}_{\odot}$. An important parameter of the model is the heating efficiency, giving the fraction of the wind mechanical energy that is converted into heat of the hot cluster winds. In the case of a low heating efficiency $(\sim 5 \%)$ the freefall time is shorter compared to the self-shielding time, forming centrally concentrated second stellar generation. On the other hand, when the heating efficiency is high $(\sim 30 \%)$ the gas streams become self shielded before they reach the cluster center, resulting in a second generation cluster with a radius similar to that of the first stellar generation.

\section{References}

D'Ercole, A., D'Antona, F., Carini, R., et al.. 2012, MNRAS, 423, 1521

Gratton, R. G., Carretta, E., \& Bragaglia, A. 2012, A\& ARv, 20, 50

Hempel, M., Sarajedini, A., Anderson, J., et al. 2014, ApJS, 211, 1

Krause, M., Charbonnel, C., Decressin, T., Meynet, G., \& Prantzos, N. 2013, A $\& A$, 552, 121

Palouš, J., Wünsch, R., Martínez-Gonzalez, S, Hueyotl-Zahuantitla, F., Silich, S., \& TenorioTagle, G. 2013, ApJ, 772, 128

Piotto, G., Milione, A. P., Anderson, J., et al. 2012, ApJ, 760, 39

Tenorio-Tagle, G., Wünsch, R., Silich, S., \& Palouš, J. 2007, ApJ, 658, 1196

Wünsch, R., Silich, S., Palouš, J., Tenorio-Tagle, G., \& Muñoz-Tuñon, C. 2011, ApJ, 740, 75 